\title{
Food Habits, Weight Status and Metabolic Risk Factors in a Group of Adults in Tehran
}

\author{
Razieh Anari ${ }^{1}$, Maryam Amini $^{2} *$, Tirang R Neyestani ${ }^{2}$ \\ 1- Ph.D. Candidate in Nutrition Research, Department of Nutrition Research, Faculty of Nutrition Sciences and Food Technology, National Nutrition and \\ Food Technology Research Institute, Shahid Beheshti University of Medical Sciences, Tehran, Iran \\ 2- Department of Nutrition Research, Faculty of Nutrition Sciences and Food Technology, National Nutrition and Food Technology Research Institute, \\ Shahid Beheshti University of Medical Sciences, Tehran, Iran
}

\section{A B S T R A C T}

Background and Objectives: This study was carried out to assess food habits and their associations with weight status and metabolic risk factors in adults in Tehran, Capital of Iran.

Materials and Methods: A cross-sectional survey was conducted in a sample of adults ( $n=227)$ over 18 years old residing in Tehran. Demographic, anthropometric and food frequency questionnaires were filled out for all of the participants. Food habits were recorded using dietary habit questionnaires. Serum concentrations of glucose and lipids (total cholesterol [TC], triglycerides [TG] and high-density lipoprotein-cholesterol [HDL-C]) were analyzed from fasting blood samples.

Results: The mean body mass index (BMI) was $28.52 \mathrm{~kg} / \mathrm{m}^{2}$ and a minority of the participants (22.2\%) had normal BMI. The participants rarely used table salt, fast food and crispy foods, dined at restaurants and had two snacks, three or more meals a day, five or more breakfast episodes a week and regular meals. They used vegetable oils for cooking and frying and/or cooking oil for frying. Drivers $(p<0.001)$ and less educated participants $(p=0.012)$ were susceptible to overweight. Positive associations were found between the BMI and the participant age $\left(\mathrm{r}_{\mathrm{s}}=0.28, p<0.001\right)$ and serum glucose $\left(\mathrm{r}_{\mathrm{s}}=0.22\right.$, $p=0.005)$, TG $\left(\mathrm{r}_{\mathrm{s}}=0.34, p<0.001\right)$ and TC $\left(\mathrm{r}_{\mathrm{s}}=0.36, p<0.001\right)$. Participants with normal weights had regular meals $(p=0.034)$ and frequent snacks $(p=0.048)$. Overweight and obese participants had a higher rate of dyslipidemia (serum $\mathrm{TG} \geq 150 \mathrm{mg} / \mathrm{dL})$, compared to that of the normal weight participants (18.0 and $35.0 \% \mathrm{vs} .7 .9 \%, p=0.004)$.

Conclusions: The present participants had relatively healthy food habits, except for the order of meals and saturated fat consumptions. This study confirms the associations between overweight/obesity and dyslipidemia. Nutrition education with emphases on regular meals and frequent snacks (as protective habits against obesity) is recommended, especially for susceptible groups.

Keywords: Food habits, Adults, Metabolic risk factors, Overweight

\section{Introduction}

In recent decades, shifts in suboptimal lifestyle, especially unhealthy dietary habits, along with socioeconomic changes have been seen in the Middle East $(1,2)$. Studies have suggested that poor diet may predispose people to many serious health problems, especially cardiovascular disease (CVD) and diabetes (3). Findings from a 20-year systematic assessment in Middle Eastern and North African countries have shown that suboptimal diet is the principal leading cause of mortality from cardio-metabolic disease (CMD) in the region (3) and CMD is the leading cause of death in Iran. Although food is primarily consumed in response to hunger, food selection and habits are seriously affected by external factors, including food accessibility, price, sociocultural, physiological and psychological factors as well as marketing techniques and nutritional knowledge (4). It is well documented that dietary habits, such as fast food consumption, meals frequency and meal size, are positively connected to weight gain, obesity, poor diet quality, and insulin resistance (5-9). Excessive salt (sodium) intake is also assumed to increase blood pressure and consequent heart events. Likewise, low consumptions of fruits and 
vegetables and high intake of trans fatty acids are other known risk factors for CMD in the Middle East (3). Eating frequency, as well as rate and regularity of food consumption, can cause metabolic disorders. Studies have demonstrated that irregular meal consumption and breakfast skipping are significantly associated with obesity $(10,11)$. In comparison to irregular meal pattern, regular meal episodes may increase thermic effect of food (TEF) and insulin sensitivity, which potentially is in favor of weight management and metabolic health (12).

Considering the aforementioned impacts of food habits on health and disease, it is of great importance to assess the status of the current food habits in the society and to target dietary habits with potentially harmful effects on chronic diseases, namely diabetes and CVD (13). Therefore, this study was carried out to describe food habits in a population of adults in Tehran and to show associations between food habits, weight status and metabolic risk factors.

\section{Materials and Methods}

Design: This cross-sectional study was carried out on adults aged 18 years and older, residing in Tehran $(n=$ 227), 2016. The study was approved by the National Nutrition and Food Technology Research Institute's (NNFTRI) Ethics Committee (Ethics Code: IR.SBMU.nnftri.Rec.1395.43).

Participants: Participants included $\geq 18$ year-old, nonsmoker, non-pregnant and nursing adult volunteers. The participants were living in Tehran, without any clinical situations affecting their food consumptions (such as diabetes, malignant diseases, or malabsorption syndrome), and were not on a specific diet.

General characteristics: Demographic data, such as age, gender, education level, occupation and disease history were recorded using questionnaires.

Dietary data: Usual dietary consumptions were collected using a validated 142-item dish-based food frequency questionnaire (DBFFQ) $(14,15)$. The questionnaires were subsequently analyzed using modified version of Nutritionist-IV Software for Iranians (version 4.0; Nsquared Computing, Salem, USA), and energy, macro and micronutrient compositions were extracted (16).

Food habits data: Dietary habit data were assessed using 15-item questionnaires. These included meal habits (frequency and regulation of meals consumption, and breakfast/snacks consumption), food preparation (removing visible fats from meat and skin of poultry), cooking habits (type of oil/fat used for cooking and frying, food preparation methods, weekly consumptions of fried foods), and other questions, including consumption of fast food, dining at restaurant, use of salt and supplements intake.
Anthropometric assessments: Weights were measured with minimum cloths and barefoot in standing position using portable digital scale (SECA, model 840, Maximum: $140 \mathrm{~kg}$, Germany) with accuracy of $0.1 \mathrm{~kg}$. Height was measured to the nearest $0.1 \mathrm{~cm}$ at standing position posture adjacent to the wall without shoes using non-elastic tape measure (SECA, Germany). Body mass index (BMI, $\mathrm{kg} / \mathrm{m}^{2}$ ) was determined by dividing weight $(\mathrm{kg})$ by squared height $\left(\mathrm{m}^{2}\right)$. BMI was categorized as the following: < 18.5 $\mathrm{kg} / \mathrm{m}^{2}$ as "underweight", $18.5-24.9 \mathrm{~kg} / \mathrm{m}^{2}$ as "normal weight", $25-29.9 \mathrm{~kg} / \mathrm{m}^{2}$ as "overweight", and $\geq 30 \mathrm{~kg} / \mathrm{m}^{2}$ as "obese"(17).

Biochemical assessments: Concentrations of glucose and lipids, including total cholesterol [TC], triglycerides [TG], and high-density lipoprotein-cholesterol [HDL-C]), were assessed in serum samples after 12 hours fasting using Pars Azmun kits (Pars Azmun, Karaj, Iran). Values were categorized as abnormal when fasting blood sugar (FBS) $\geq$ $100 \mathrm{mg} / \mathrm{dL}, \mathrm{HDL}-\mathrm{C}<50 \mathrm{mg} / \mathrm{dL}$ in women and $<40 \mathrm{mg} / \mathrm{dL}$ in men, $\mathrm{TG} \geq 150 \mathrm{mg} / \mathrm{dL}$, and cholesterol $\geq 200 \mathrm{mg} / \mathrm{dL}$ ( 18 , 19).

Statistical analyses: Statistical analyses were performed using SPSS Software (version 21.0, SPSS Inc., Chicago, IL, USA). Data were reported as mean \pm SD (standard deviation) and percentage. Categorical variables were compared using chi-square test, binary logistic regression, one-way analysis of variance (ANOVA) and Kruskal-Walis tests with $95 \%$ confidence interval. The $p$-values less than 0.05 were reported as significant.

\section{Results}

Totally, 227 adults (47.6\% men) were participated in the study. Demographic data, dietary energy and macronutrient intakes and biochemical measures are shown in Table 1. Only $22.2 \%$ of the participants had normal BMI. Table 2 shows the associations between weight status, demographic data and food habits of the participants. There was a slight positive correlation between age and BMI $\left(r_{s}=0.275\right.$, $p<0.001)$. The mean age of participants differed significantly among the BMI subgroups $(p=0.001$, Table 2 ). Despite similar energy and macronutrient intakes, obese and overweight participants were averagely 7.73 and 6.20 years older than normal weight participants $(p=0.001$ and $p=0.006$, respectively, Table 2). Most of the overweight and obese participants were drivers $(p<0.001)$, and most of the normal weight participants had academic degree $(p=0.012)$. Regularity of meals and frequency of snack consumption were the only dissimilar habits within various BMI subgroups $(p=0.005)$. As shown in Table 2, overweight and obese participants had lower snack occasions per day $(p=0.048)$. Participants who always or often consumed regular meals had significantly lower rates of obesity or overweight ( $p=0.034)$. 
Table 1. Demographic data of a sample of adults in Tehran $(n=227), 2016$

\begin{tabular}{|c|c|}
\hline variable & Value $\mathrm{n}(\%)$ \\
\hline Participants & $227(100)$ \\
\hline Male, & $108(47.6)$ \\
\hline Female & $119(52.4)$ \\
\hline Total & 227 \\
\hline \multicolumn{2}{|l|}{ Education, } \\
\hline Under diploma & $49(21.6)$ \\
\hline High School diploma & $107(47.1)$ \\
\hline Academic degree & $71(31.3)$ \\
\hline Total & $227(100)$ \\
\hline \multicolumn{2}{|l|}{ Occupation, } \\
\hline Driver & $84(37.3)$ \\
\hline Clerk & $43(19.1)$ \\
\hline College student & $22(9.8)$ \\
\hline Housekeeping & $51(22.7)$ \\
\hline Other & $25(11.1)$ \\
\hline Total & $225(100)$ \\
\hline \multicolumn{2}{|l|}{ Weight status, } \\
\hline Normal & $49(22.2)$ \\
\hline Overweight & $92(41.6)$ \\
\hline Obesity & $80(36.2)$ \\
\hline Total & $221(100)$ \\
\hline Variable(unit) & Mean \pm SD \\
\hline Age (yr) & $42.18 \pm 11.63$ \\
\hline $\mathrm{n}$ & 227 \\
\hline BMI $(\mathrm{kg} / \mathrm{m} 2)$ & $28.52 \pm 4.66$ \\
\hline $\mathrm{n}$ & 226 \\
\hline $\mathrm{CHO}(\mathrm{gr} / \mathrm{d})$ & $571.30 \pm 247.97$ \\
\hline $\mathrm{n}$ & 222 \\
\hline $\operatorname{Pr}(\mathrm{gr} / \mathrm{d})$ & $112.21 \pm 46.41$ \\
\hline $\mathrm{n}$ & 220 \\
\hline Fat $(\mathrm{gr} / \mathrm{d})$ & $110.85 \pm 47.68$ \\
\hline $\mathrm{n}$ & 222 \\
\hline $\mathrm{FBS}(\mathrm{mg} / \mathrm{dL})$ & $85.11 \pm 9.48$ \\
\hline $\mathrm{n}$ & 162 \\
\hline $\mathrm{TG}(\mathrm{mg} / \mathrm{dL})$ & $126.95 \pm 57.06$ \\
\hline $\mathrm{n}$ & 161 \\
\hline Chol (mg/dL) & $187.84 \pm 33.45$ \\
\hline $\mathrm{n}$ & 164 \\
\hline HDL (mg/dL) & $50.45 \pm 10.71$ \\
\hline $\mathrm{n}$ & 164 \\
\hline
\end{tabular}

$B M I$ body mass index, $C H O$ carbohydrate, $C h o l$ cholesterol, $F B S$ fasting blood sugar, $H D L$ high density lipoprotein, $\operatorname{Pr}$ protein, $T G$ triglyceride. n indicates total number of cases. Values are Mean \pm SD except stated. $P$ $<0.05$ is significant.

When this association was adjusted by gender, it was only significant in males ( $p=0.044$ for males vs. $p=0.094$ for females). Analyses revealed that obese participants were less likely to use multivitamin/mineral supplements than normal weight participants $(p=0.058)$. The participants who had three or more meals a day possibly had more weekly breakfast episodes (five or more per week) compared to others $(91.2 \%$ vs.52.0\%, $p<0.001)$. Our findings showed that $23.4 \%$ and $2.5 \%$ of the participants used hydrogenated vegetable oil and/or animal fat for cooking and frying, respectively. No difference was observed among BMI subgroups regarding type of consumed fat/oil for frying or cooking. Participants in all BMI categories mostly used frying oil in combination with cooking oil for frying. Furthermore, hydrogenated vegetable oils or animal fats were mostly used in cooking process, regardless of BMI status (Table 2).

Nearly one-third $(34.4 \%)$ of the participants consumed supplements routinely. Only $11.9 \%$ of the participants used vitamin D supplement, $92.6 \%$ of them had a daily to monthly supplement intake. Consumptions of other supplements were as the following: $4.4 \%$ for vitamin $\mathrm{C}$ ( $90 \%$ daily to monthly use), $4.0 \%$ for vitamin A $(33.3 \%$ daily and $66.7 \%$ weekly use), and $3.1 \%$ for zinc supplements ( $57.1 \%$ daily and $42.9 \%$ weekly use). Other frequent food habits included never to rare use of table salt $(68.4 \%)$, rare consumption of fast food (i.e. $\leq 1$ time/month) (43.1\%), 2 snacks/day (42.3\%), $\geq 5$ breakfast episodes/week (78.0\%), $\geq 3$ meals/day (66.4\%), always regular meals $(39.6 \%)$, rare dining at restaurant $(\leq 1$ time/month) (51.8\%), use of vegetable oils for cooking (except saturated and frying oils, 59.6\%), rare consumption of crispy foods ( $<1$ time/month) $(63.1 \%)$, and use of frying or cooking oils for frying (76.4\%). Biochemical analyses demonstrated that $5.6,21.7,23.8$ and $31.1 \%$ of the participants had abnormal concentrations of FBS, TG, HDL-C and TC, respectively. Significant associations were seen between HDL-C and frying method and also TC and regulation of meals, consumption of crispy fried foods, dining at restaurant and removing trimming fats from red meat. Deep frying rather than Sauté frying elevated the chance of abnormal HDL-C concentrations (binary logistic regression, $\mathrm{B}=6.51, p=0.024)$. The $\mathrm{TC}$ levels were associated with regular meals consumption $(\mathrm{B}=-5.88, p=$ $0.011),<1$ time/m Crispy fried foods $(\mathrm{B}=5.29, p=$ $0.044)$, monthly dining at restaurant $(\mathrm{B}=3.27, p=0.033)$, and partial trimming of visible fats from red meat $(\mathrm{B}=-$ $6.69, p=0.048)$. The BMI was positively correlated with FBS $\left(r_{\mathrm{s}}=0.223, p=0.005\right)$, TG $\left(\mathrm{r}_{\mathrm{s}}=0.342, p<0.001\right)$ and $\mathrm{TC}\left(\mathrm{r}_{\mathrm{s}}=0.359, p<0.001\right)$, but not with serum HDL-C $\left(\mathrm{r}_{\mathrm{s}}=\right.$ $-0.130, p=0.099)$. However, the association of energy and macronutrient intakes with FBS, TG and HDL-C abnormalities was not statistically significant. There was a noteworthy association between BMI categories and TG status ( $p=0.004$, Table 3 ). Logistic regression revealed that obese participants had higher odds of abnormal TG values $(\mathrm{OR}=6.28,95 \% \mathrm{CI}=1.72-22.89, p=0.005)$. This association remained significant even after adjusting for age, energy and macronutrient intakes $(\mathrm{OR}=5.28,95 \% \mathrm{CI}=1.37-20.35$, $p=0.016$ ). However, after further adjustments for other confounding factors (e.g., job and education), this association was near significant (binary logistic regression, $p=0.052$ ). 
Razieh Anari, et al: Food habits, weight status \& metabolic risk factors

Table 2. Association of BMI and food habits in a sample of adults in Tehran $(n=227), 2016$

\begin{tabular}{|c|c|c|c|c|}
\hline \multirow[t]{2}{*}{ Variable } & \multicolumn{3}{|c|}{ BMI $\left(\mathrm{kg} / \mathrm{m}^{2}\right)$} & \multirow[t]{2}{*}{ p-value } \\
\hline & $\begin{array}{c}18.5-24.9 \\
\text { (Normal weight) }\end{array}$ & $\begin{array}{c}25-29.9 \\
\text { (overweight) }\end{array}$ & $\begin{array}{c}\geq 30 \\
\text { (obesity) }\end{array}$ & \\
\hline $\begin{array}{l}\text { Age (year) } \\
\text { Total }\end{array}$ & $\begin{array}{c}37.00 \pm 11.73 \\
50 \\
\end{array}$ & $\begin{array}{c}43.08 \pm 11.48 \\
94\end{array}$ & $\begin{array}{c}44.68 \pm 10.68 \\
79 \\
\end{array}$ & $0.001 *$ \\
\hline $\begin{array}{l}\text { Sex } \\
\text { Male } \\
\text { Female } \\
\text { Total }\end{array}$ & $\begin{array}{c}24(48.0 \%) \\
26(52.0 \%) \\
50(100.0 \%)\end{array}$ & $\begin{array}{l}51(54.3 \%) \\
43(45.7 \%) \\
94(100.0 \%)\end{array}$ & $\begin{array}{c}31(39.2 \%) \\
48(60.8 \%) \\
79(100.0 \%)\end{array}$ & 0.143 \\
\hline $\begin{array}{l}\text { Education } \\
\text { under diploma } \\
\text { diploma } \\
\text { academic degree } \\
\text { Total } \\
\end{array}$ & $\begin{array}{l}10(20.0 \%) \\
17(34.0 \%) \\
23(46.0 \%) \\
50(100.0 \%) \\
\end{array}$ & $\begin{array}{l}16(17.0 \%) \\
47(50.0 \%) \\
31(33.0 \%) \\
94(100.0 \%) \\
\end{array}$ & $\begin{array}{c}23(29.1 \%) \\
41(51.9 \%) \\
15(19.0 \%) \\
79(100.0 \%) \\
\end{array}$ & $\begin{array}{c}0.012^{*} \\
(\mathrm{male}=0.280 \\
\left.\text { female }=0.041^{*}\right)\end{array}$ \\
\hline $\begin{array}{l}\text { Occupation } \\
\text { driver } \\
\text { others } \\
\text { clerk } \\
\text { college student } \\
\text { Total } \\
\end{array}$ & $\begin{array}{c}6(12.0 \%) \\
23(46.0 \%) \\
9(18.0 \%) \\
12(24.0 \%) \\
50(100.0 \%) \\
\end{array}$ & $\begin{array}{c}40(43.5 \%) \\
27(29.3 \%) \\
20(21.7 \%) \\
5(5.4 \%) \\
92(100.0 \%) \\
\end{array}$ & $\begin{array}{l}36(45.6 \%) \\
25(31.6 \%) \\
14(17.7 \%) \\
4(5.1 \%) \\
79(100.0 \%) \\
\end{array}$ & $\begin{array}{c}<0.001^{* *} \\
\left(\text { male }<0.001^{* *} ;\right. \\
\text { female }=0.063)\end{array}$ \\
\hline $\begin{array}{l}\text { Energy }(\mathrm{kcal} / \mathrm{d}) \\
\text { Total }\end{array}$ & $\begin{array}{c}3546.68 \pm 1430.66 \\
50\end{array}$ & $\begin{array}{c}3784.97 \pm 1548.70 \\
91\end{array}$ & $\begin{array}{c}3659.84 \pm 1654.08 \\
78\end{array}$ & 0.622 \\
\hline $\begin{array}{l}\text { Carbohydrate }(\mathrm{g} / \mathrm{d}) \\
\text { Total }\end{array}$ & $\begin{array}{c}537.75 \pm 212.06 \\
50 \\
\end{array}$ & $\begin{array}{c}597.85 \pm 270.48 \\
92 \\
\end{array}$ & $\begin{array}{c}562.45 \pm 245.48 \\
77 \\
\end{array}$ & 0.552 \\
\hline $\begin{array}{l}\text { Protein }(\mathrm{g} / \mathrm{d}) \\
\text { Total }\end{array}$ & $\begin{array}{c}107.62 \pm 39.39 \\
49\end{array}$ & $\begin{array}{c}113.08 \pm 42.33 \\
90\end{array}$ & $\begin{array}{c}109.96 \pm 48.14 \\
76\end{array}$ & 0.588 \\
\hline $\begin{array}{l}\text { Fat }(\mathrm{g} / \mathrm{d}) \\
\text { Total }\end{array}$ & $\begin{array}{c}112.18 \pm 53.89 \\
50 \\
\end{array}$ & $\begin{array}{c}114.42 \pm 44.76 \\
91\end{array}$ & $\begin{array}{c}105.89 \pm 47.70 \\
78 \\
\end{array}$ & 0.293 \\
\hline $\begin{array}{l}\text { No. of meals taken per day } \\
<3 \\
\geq 3 \\
\text { Total }\end{array}$ & $\begin{array}{c}13(26.0 \%) \\
37(74.0 \%) \\
50(100.0 \%)\end{array}$ & $\begin{array}{c}30(31.9 \%) \\
64(68.1 \%) \\
94(100.0 \%)\end{array}$ & $\begin{array}{l}31(39.7 \%) \\
47(60.3 \%) \\
78(100.0 \%)\end{array}$ & $\begin{array}{c}0.254 \\
(\mathrm{male}=0.285 \\
\text { female }=0.692)\end{array}$ \\
\hline $\begin{array}{l}\text { No. of breakfasts taken per week } \\
\leq 4 \\
\geq 5 \\
\text { Total }\end{array}$ & $\begin{array}{l}10(20.0 \%) \\
40(80.0 \%) \\
50(100.0 \%) \\
\end{array}$ & $\begin{array}{c}17(18.5 \%) \\
75(81.5 \%) \\
92(100.0 \%) \\
\end{array}$ & $\begin{array}{r}21(27.3 \%) \\
56(72.7 \%) \\
77(100.0 \%) \\
\end{array}$ & $\begin{array}{c}0.362 \\
(\text { male }=0.594 \\
\text { female }=0.268)\end{array}$ \\
\hline $\begin{array}{l}\text { Frequency of regular meals } \\
\text { Never or Sometimes } \\
\text { Often or Always } \\
\text { Total }\end{array}$ & $\begin{array}{c}13(26.5 \%) \\
36(73.5 \%) \\
49(100.0 \%)\end{array}$ & $\begin{array}{c}31(33.7 \%) \\
61(66.3 \%) \\
92(100.0 \%)\end{array}$ & $\begin{array}{c}37(48.1 \%) \\
40(51.9 \%) \\
77(100.0 \%)\end{array}$ & $\begin{array}{c}0.034^{*} \\
\left(\text { male }=0.044^{*}\right. \\
\text { female }=0.094)\end{array}$ \\
\hline $\begin{array}{l}\text { No. of Snacks eaten per day } \\
1 \\
2 \\
3-5 \\
\text { Total }\end{array}$ & $\begin{array}{l}15(30.6 \%) \\
16(32.7 \%) \\
18(36.7 \%) \\
49(100.0 \%) \\
\end{array}$ & $\begin{array}{l}33(35.9 \%) \\
42(45.7 \%) \\
17(18.5 \%) \\
92(100.0 \%) \\
\end{array}$ & $\begin{array}{l}30(40.0 \%) \\
34(45.3 \%) \\
11(14.7 \%) \\
75(100.0 \%) \\
\end{array}$ & $\begin{array}{c}0.048^{*} \\
(\text { male }=0.389 \\
\text { female }=0.138)\end{array}$ \\
\hline $\begin{array}{l}\text { Trimming of visible fat from meat before } \\
\text { cooking } \\
\text { I do not eat meat at all } \\
\text { I trim most of it } \\
\text { I trim little of it } \\
\text { I do not trim it } \\
\text { Total }\end{array}$ & $\begin{array}{c}2(4.1 \%) \\
36(73.5 \%) \\
2(4.1 \%) \\
9(18.4 \%) \\
49(100.0 \%) \\
\end{array}$ & $\begin{array}{c}2(2.1 \%) \\
63(67.0 \%) \\
13(13.8 \%) \\
16(17.0 \%) \\
94(100.0 \%) \\
\end{array}$ & $\begin{array}{c}5(6.5 \%) \\
49(63.6 \%) \\
6(7.8 \%) \\
17(22.1 \%) \\
77(100.0 \%) \\
\end{array}$ & $\begin{array}{c}0.362 \\
(\text { male }=0.615 \\
\text { female }=0.362)\end{array}$ \\
\hline $\begin{array}{l}\text { Removal of poultry skin before cooking } \\
\text { Never } \\
\text { Sometimes } \\
\text { Often } \\
\text { Always } \\
\text { Total }\end{array}$ & $\begin{array}{c}3(6.0 \%) \\
1(2.0 \%) \\
1(2.0 \%) \\
45(90.0 \%) \\
50(100.0 \%)\end{array}$ & $\begin{array}{c}1(1.1 \%) \\
3(3.2 \%) \\
2(2.1 \%) \\
88(93.6 \%) \\
94(100.0 \%)\end{array}$ & $\begin{array}{c}0(0.0 \%) \\
2(2.6 \%) \\
3(3.9 \%) \\
72(93.5 \%) \\
77(100.0 \%)\end{array}$ & $\begin{array}{c}0.288 \\
(\text { male }=0.445 \\
\text { female }=0.296)\end{array}$ \\
\hline
\end{tabular}


Razieh Anari, et al: Food habits, weight status \& metabolic risk factors

\begin{tabular}{|c|c|c|c|c|}
\hline \multirow[t]{2}{*}{ Variable } & \multicolumn{3}{|c|}{ BMI $\left(\mathrm{kg} / \mathrm{m}^{2}\right)$} & \multirow[t]{2}{*}{ p-value } \\
\hline & $\begin{array}{c}18.5-24.9 \\
\text { (Normal weight) }\end{array}$ & $\begin{array}{c}25-29.9 \\
\text { (overweight) }\end{array}$ & $\begin{array}{c}\geq 30 \\
\text { (obesity) }\end{array}$ & \\
\hline $\begin{array}{l}\text { No. of fried food eaten per week } \\
\text { Never } \\
1-3 \\
4-6 \\
\text { Every day } \\
\text { Total } \\
\end{array}$ & $\begin{array}{c}1(2.0 \%) \\
35(70.0 \%) \\
10(20.0 \%) \\
4(8.0 \%) \\
50(100.0 \%) \\
\end{array}$ & $\begin{array}{c}4(4.3 \%) \\
73(77.7 \%) \\
16(17.0 \%) \\
1(1.1 \%) \\
94(100.0 \%) \\
\end{array}$ & $\begin{array}{c}7(9.2 \%) \\
53(69.7 \%) \\
14(18.4 \%) \\
2(2.6 \%) \\
76(100.0 \%) \\
\end{array}$ & $\begin{array}{c}0.176 \\
(\text { male }=0.158 \\
\text { female }=0.259)\end{array}$ \\
\hline $\begin{array}{l}\text { Method of frying } \\
\text { Deep } \\
\text { Superficial } \\
\text { Sauté } \\
\text { Total }\end{array}$ & $\begin{array}{l}4(8.7 \%) \\
33(71.7 \%) \\
9(19.6 \%) \\
46(100.0 \%) \\
\end{array}$ & $\begin{array}{c}8(9.2 \%) \\
63(72.4 \%) \\
16(18.4 \%) \\
87(100.0 \%) \\
\end{array}$ & $\begin{array}{c}8(11.3 \%) \\
51(71.8 \%) \\
12(16.9 \%) \\
71(100.0 \%)\end{array}$ & $\begin{array}{c}0.985 \\
(\text { male }=0.894 ; \\
\text { female }=0.558)\end{array}$ \\
\hline $\begin{array}{l}\text { Type of oil/fat used for frying } \\
\text { Frying oil or combination of cooking \& } \\
\text { frying oil }\end{array}$ & $37(77.1 \%)$ & $58(71.6 \%)$ & $53(80.3 \%)$ & $\begin{array}{c}0.523 \\
(\text { male }=0.074 \\
\text { female }=0.732)\end{array}$ \\
\hline $\begin{array}{l}\text { Olive or other veg. oils (including cooking } \\
\text { oil, hydrogenated, etc.) or combination of } \\
\text { them } \\
\text { Hydrogenated veg. oil, animal fat (rump, } \\
\text { ghee, butter, etc.) or combination of any veg. } \\
\text { oil \& animal } \\
\text { Total }\end{array}$ & $\begin{array}{c}0(0.0 \%) \\
48(100.0 \%)\end{array}$ & $\begin{array}{r}3(3.7 \%) \\
81(100.0 \%)\end{array}$ & $\begin{array}{c}11(16.7 \%) \\
2(3.0 \%) \\
66(100.0 \%)\end{array}$ & \\
\hline $\begin{array}{l}\text { Type of oil/fat used for cooking } \\
\text { Frying oil }\end{array}$ & $3(8.1 \%)$ & $8(11.0 \%)$ & $9(15.5 \%)$ & $\begin{array}{c}0.963 \\
\text { (male }=0.994 ; \\
\text { female }=0.684)\end{array}$ \\
\hline $\begin{array}{l}\text { Other veg. oil (olive, corn, soy, sunflower, } \\
\text { sesame, etc.) }\end{array}$ & $23(62.2 \%)$ & $43(58.9 \%)$ & $34(58.6 \%)$ & \\
\hline $\begin{array}{l}\text { Hydrogenated veg. oil, animal fat (rump, } \\
\text { ghee, butter, etc.) or combination of them }\end{array}$ & $9(24.3 \%)$ & $18(24.7 \%)$ & $12(20.7 \%)$ & \\
\hline $\begin{array}{l}\text { Combination of oils/fats (frying, olive, corn, } \\
\text { soy, hydrogenated, animal, etc.) }\end{array}$ & $2(5.4 \%)$ & $4(5.5 \%)$ & & \\
\hline Total & $37(100.0 \%)$ & $73(100.0 \%)$ & $58(100.0 \%)$ & \\
\hline $\begin{array}{l}\text { Frequency of salt use on table } \\
\text { Always } \\
\text { Sometimes or Often } \\
\text { Never or rarely } \\
\text { Total }\end{array}$ & $\begin{array}{c}8(16.3 \%) \\
9(18.4 \%) \\
32(65.3 \%) \\
49(100.0 \%)\end{array}$ & $\begin{array}{l}14(14.9 \%) \\
10(10.6 \%) \\
70(74.5 \%) \\
94(100.0 \%)\end{array}$ & $\begin{array}{l}9(11.5 \%) \\
20(25.6 \%) \\
49(62.8 \%) \\
78(100.0 \%)\end{array}$ & $\begin{array}{c}0.138 \\
(\text { male }=0.191 \\
\text { female }=0.516)\end{array}$ \\
\hline $\begin{array}{l}\text { No. of Crispy fried foods eaten out } \\
\text { Never } \\
<1 / \text { month } \\
1 / \text { month } \\
>1 / \text { month } \\
\text { Total } \\
\end{array}$ & $\begin{array}{c}27(54.0 \%) \\
2(4.0 \%) \\
13(26.0 \%) \\
8(16.0 \%) \\
50(100.0 \%) \\
\end{array}$ & $\begin{array}{c}49(52.7 \%) \\
9(9.7 \%) \\
16(17.2 \%) \\
19(20.4 \%) \\
93(100.0 \%) \\
\end{array}$ & $\begin{array}{c}42(53.8 \%) \\
11(14.1 \%) \\
15(19.2 \%) \\
10(12.8 \%) \\
78(100.0 \%) \\
\end{array}$ & $\begin{array}{c}0.431 \\
(\text { male }=0.796, \\
\text { female }=0.645)\end{array}$ \\
\hline $\begin{array}{l}\text { Frequency of fast food consumption } \\
\text { Never } \\
\text { Weekly } \\
\text { Monthly } \\
\text { Rarely (<1 time/Mo.) } \\
\text { Total }\end{array}$ & $\begin{array}{l}20(41.7 \%) \\
7(14.6 \%) \\
18(37.5 \%) \\
3(6.3 \%) \\
48(100.0 \%) \\
\end{array}$ & $\begin{array}{l}40(42.6 \%) \\
13(13.8 \%) \\
28(29.8 \%) \\
13(13.8 \%) \\
94(100.0 \%)\end{array}$ & $\begin{array}{c}40(51.9 \%) \\
5(6.5 \%) \\
26(33.8 \%) \\
6(7.8 \%) \\
77(100.0 \%)\end{array}$ & $\begin{array}{c}0.360 \\
\text { (male }=0.268 \\
\text { female }=0.206\end{array}$ \\
\hline $\begin{array}{l}\text { Eating food at restaurant } \\
\text { Weekly } \\
\text { Monthly } \\
\text { Rarely }(<1 / \mathrm{mo} .) \\
\text { Never } \\
\text { Total }\end{array}$ & $\begin{array}{c}22(44.0 \%) \\
6(12.0 \%) \\
18(36.0 \%) \\
4(8.0 \%) \\
50(100.0 \%) \\
\end{array}$ & $\begin{array}{c}27(29.3 \%) \\
13(14.1 \%) \\
40(43.5 \%) \\
12(13.0 \%) \\
92(100.0 \%) \\
\end{array}$ & $\begin{array}{c}24(30.8 \%) \\
15(19.2 \%) \\
27(34.6 \%) \\
12(15.4 \%) \\
78(100.0 \%) \\
\end{array}$ & $\begin{array}{c}0.445 \\
(\text { male }=0.277 \\
\text { female }=0.526)\end{array}$ \\
\hline $\begin{array}{l}\text { Use of multivitamin-mineral supplements } \\
\text { No } \\
\text { Yes } \\
\text { Total }\end{array}$ & $\begin{array}{c}26(54.2 \%) \\
22(45.8 \%) \\
48(100.0 \%)\end{array}$ & $\begin{array}{l}55(61.1 \%) \\
35(38.9 \%) \\
90(100.0 \%) \\
\end{array}$ & $\begin{array}{c}57(74.0 \%) \\
20(26.0 \%) \\
77(100.0 \%)\end{array}$ & $\begin{array}{c}0.058 \\
(\text { male }=0.356 \\
\text { female }=0.062)\end{array}$ \\
\hline
\end{tabular}

Shapiro-Wilk test used to check normality. Analyses were done after removing outliers. Chi-square, One-way ANOVA and Kruskal-Wallis tests were used to analyze data.

$* \mathrm{p}<0.05$ 
Razieh Anari, et al: Food habits, weight status \& metabolic risk factors

Table 3. Biochemical analyses and weight status in a sample of adults in Tehran $(n=227), 2016$

\begin{tabular}{|c|c|c|c|c|}
\hline \multirow[t]{2}{*}{ Factor } & \multicolumn{3}{|c|}{ BMI } & \multirow[t]{2}{*}{ p value ${ }^{i}$} \\
\hline & $\begin{array}{c}18.5-24.9 \\
\text { (Normal weight) }\end{array}$ & $\begin{array}{c}25-29.9 \\
\text { (overweight) }\end{array}$ & $\begin{array}{c}\geq 30 \\
\text { (obesity) }\end{array}$ & \\
\hline $\begin{array}{l}\text { FBS, mg/dL } \\
<100 \\
\geq 100 \\
\text { Total }\end{array}$ & $\begin{array}{c}37(97.4 \%) \\
1(2.6 \%) \\
38(100.0 \%)\end{array}$ & $\begin{array}{c}60(93.8 \%) \\
4(6.3 \%) \\
64(100.0 \%)\end{array}$ & $\begin{array}{c}54(93.1 \%) \\
4(6.9 \%) \\
58(100.0 \%)\end{array}$ & $\begin{array}{l}0.649 \\
(\text { male }=0.201 ; \\
\text { female }=0.053)\end{array}$ \\
\hline $\begin{array}{l}\text { Crude OR } \\
\text { Adjusted } \mathrm{OR}^{\mathrm{ii}}\end{array}$ & $\begin{array}{l}1 \\
1\end{array}$ & $\begin{array}{l}2.47(\mathrm{p}=0.427) \\
1.99(\mathrm{p}=0.495)\end{array}$ & $\begin{array}{l}2.74(p=0.376) \\
2.24(p=0.495)\end{array}$ & \\
\hline $\begin{array}{l}\text { TG, mg/dL } \\
<150 \\
\geq 150 \\
\text { Total }\end{array}$ & $\begin{array}{c}35(92.1 \%) \\
3(7.9 \%) \\
38(100.0 \%)\end{array}$ & $\begin{array}{l}50(82.0 \%) \\
11(18.0 \%) \\
61(100.0 \%)\end{array}$ & $\begin{array}{l}39(65.0 \%) \\
21(35.0 \%) \\
60(100.0 \%)\end{array}$ & $\begin{array}{l}0.004 * \\
(\text { male }=0.050 \\
\text { female }=0.017 *)\end{array}$ \\
\hline $\begin{array}{l}\text { Crude OR } \\
\text { Adjusted OR }\end{array}$ & $\begin{array}{l}1 \\
1\end{array}$ & $\begin{array}{l}2.57(\mathrm{p}=0.170) \\
2.30(\mathrm{p}=0.251)\end{array}$ & $\begin{array}{l}6.28(\mathrm{p}=0.005)^{*} \\
5.28(\mathrm{p}=0.016)^{*}\end{array}$ & \\
\hline $\begin{array}{l}\mathrm{HDL}-\mathrm{C}, \mathrm{mg} / \mathrm{dL} \\
\geq 40 \mathrm{~m} \text { or } \geq 50 \mathrm{f} \\
<40 \mathrm{~m} \text { or }<50 \mathrm{f} \\
\text { Total }\end{array}$ & $\begin{array}{c}35(92.1 \%) \\
3(7.9 \%) \\
38(100.0 \%)\end{array}$ & $\begin{array}{c}49(76.6 \%) \\
15(23.4 \%) \\
64(100.0 \%)\end{array}$ & $\begin{array}{c}39(65.0 \%) \\
21(35.0 \%) \\
60(100.0 \%)\end{array}$ & $\begin{array}{l}0.009 * \\
(\text { male }=0.305 ; \\
\text { female }=0.020 *)\end{array}$ \\
\hline $\begin{array}{l}\text { Crude OR } \\
\text { Adjusted } \mathrm{OR}^{\mathrm{ii}}\end{array}$ & $\begin{array}{l}1 \\
1\end{array}$ & $\begin{array}{c}3.57(\mathrm{p}=0.057) \\
4.28(\mathrm{p}=0.038)^{*}\end{array}$ & $\begin{array}{l}6.28(\mathrm{p}=0.005)^{*} \\
8.01(\mathrm{p}=0.003)^{*}\end{array}$ & \\
\hline $\begin{array}{l}\text { TC, mg/dL } \\
<200 \\
\geq 200 \\
\text { Total }\end{array}$ & $\begin{array}{c}32(84.2 \%) \\
6(15.8 \%) \\
38(100.0 \%)\end{array}$ & $\begin{array}{l}47(73.4 \%) \\
17(26.6 \%) \\
64(100.0 \%)\end{array}$ & $\begin{array}{l}33(55.0 \%) \\
27(45.0 \%) \\
60(100.0 \%)\end{array}$ & $\begin{array}{l}0.006 * \\
(\text { male }=0.023 * ; \\
\text { female }=0.125)\end{array}$ \\
\hline $\begin{array}{l}\text { Crude OR } \\
\text { Adjusted OR }\end{array}$ & $\begin{array}{l}1 \\
1\end{array}$ & $\begin{array}{l}1.93(\mathrm{p}=0.213) \\
1.46(\mathrm{p}=0.505)\end{array}$ & $\begin{array}{l}4.36(\mathrm{p}=0.004) * \\
3.48(\mathrm{p}=0.023)^{*}\end{array}$ & \\
\hline
\end{tabular}

\section{Discussion}

In general, it is not precisely clear which food habits may increase or decrease risks of obesity and other metabolic disorders. For example, the Adventist Health Study-2 over 30 years proposed that habits like less frequent meals, no snacking, breakfast consumption, and consumption of the largest meal in the morning may prevent weight gain in long term (20). However, other studies have suggested irregular $(10,11)$ and less frequent meals (21-24) as obesity risk factors. The present study demonstrated an inverse association between overweight and frequency of snacks. Some observational studies have suggested an inverse relationship between frequency of eating and adiposity (21-24). Proposed explaining mechanisms might be better appetite control $(25,26)$, improved glucose homeostasis (27-29) and increased TEF (30, 31). Furthermore, our data showed that adults consuming 3 meals/day possibly had more breakfast episodes in a week. Although no direct association was found between breakfast consumption and obesity or other metabolic disorders in this study, previous studies suggested that daily consumption of breakfast is linked to $1.9 \mathrm{~kg}$ less weight gain compared with infrequent breakfast consumption (0-3 days/week) in participants over 18 years (32). Furthermore, eating breakfast was claimed to decrease risk of diabetes and hypertension (33), and larger breakfast is associated with a significant decrement in BMI (20). Nevertheless, no data were available on breakfast size in this study.

Although no association was found between weight status and meal episodes in this study, some studies proposed that a higher eating frequency is associated with healthier lifestyle (e.g. lower smoking and alcohol use, greater physical activity and a healthier diet) (34). Middleaged population with three or less daily meals had greater chance of both general and central obesity in males, but not in females (34). In a population-based study, higher daily eating episodes were associated with lower serum cholesterol and LDL-C concentrations, as participants with four and more meals per day had $0.23 \mathrm{mmol} / \mathrm{L}$ lower TC $(p=0.01)$ and had $0.16 \mathrm{mmol} / \mathrm{L}$ lower LDL-C $(\mathrm{p}=0.06)$ 
than those with 1-2 meals per day. That proposes the possibility of serum cholesterol decline by increasing meal frequency (35). We found no association between food frequency and serum lipids. However, those with regular meals had lower TC concentrations. Previous findings suggested a positive association between irregular meal pattern and BMI (36) and obesity $(10,11)$. Based on these findings, overweight is inversely associated with meal regularity, especially in men. Greater TEF, greater insulin sensitivity, and favorable appetite changes in a regular against an irregular meal consumption may mediate a better weight control and metabolic health (12).

In the current study, an inappropriate use of saturated fats/oils for cooking were seen. Hydrogenated vegetable oils and animal fats were the second most used oils by the participants $(23.4 \%)$. These are major sources of saturated (SFAs) and trans-fatty acids (TFAs) in diet (37, 38). Several evidence have specified that TFAs increase chance of coronary heart disease (CHD) development (39, 40), which might be due to its adverse effects on serum lipids and lipoproteins $(39,41,42)$, enhanced systemic inflammation (43-46) and endothelial dysfunction (43, 45). Systematic reviews and meta-analyses have demonstrated that replacement of SFAs with poly unsaturated fatty acids (PUFAs) and monounsaturated fatty acids (MUFAs) may decrease CHD risk $(47,48)$. In previous studies, saturated fats have been connected to cancers, like gastric cancer (49). Also, a relatively high prevalence of overweight and obesity in this study was in consistence with recent findings in Iranian population (50). According to a recent investigation, non-optimal BMI was the third leading cause of CMD mortality in most Middle Eastern and North African countries (3). Obese participants had nearly six times higher odds of abnormal serum TG and higher BMIs accompanied by higher serum FBS, TG and TC levels. Nevertheless, these had no association with energy and macronutrient intakes. Previous studies have demonstrated that $60-70 \%$ of obese and $50-60 \%$ of overweight people had dyslipidemia and higher chance of diabetes (51). According to our results, older adults had higher BMIs which could be due to physical inactivity, lower lean body mass or poor food habits. Further studies are needed in this regard. In spite of high prevalence of vitamin D deficiency in Iranian population (52), a small proportion of the participants received vitamin D supplements routinely. A recent study has revealed that vitamin D deficiency is positively linked to abdominal and general obesity (53). Therefore, public health professionals should further focus on this matter.

The current study had several strengths: First, data collected using food frequency questionnaires accompanied by food habits can widely capture food intake in long-term. Second, a wide number of food habits were questioned.
We also faced some limitations: First, meal's composition and size and their possible connection with metabolic risk factors were not assessed. Second, the mediating role of the physical activity in obesity and other metabolic risk factors was ignored in this study.

\section{Conclusion}

To conclude, in spite of acceptable manner for most food habits, high consumption of saturated oils/fats as well as excessive body weight and elevated blood lipids in studied participants were seen. There was an association between obesity and dyslipidemia along with its correlation with some food habits, like snacks and regular meals consumption, which suggests that modification of food habits may mitigate the risk of non-communicable diseases, especially CVD, through weight and lipid control. It is recommended that health authorities focus on nutrition education with emphasis on regular meals and more snacks in policies regarding non-communicable disease control. Further studies should be carried out on meal composition and physical activity and underling mechanisms of food habits' effect on metabolic health. To overcome drawbacks, further investigations with greater sample sizes and longer durations are encouraged.

\section{Acknowledgement}

The authors appreciate Shahid Beheshti University of Medical Sciences, NNFTRI, for funding the study. All coordinators, interviewers and staff are appreciated. The authors would like to express their gratitude to M. Abtahi, P. Alipoor, F. Bagheri, M. Dadkhah-Piraghaj, S. Esfandiari, Dr. H. Ein-Zinab, O. Hashemkhani, H. Heidari, A. Kalayi, I. Khosroshahi, N. Lotfollahi, B. MohammadaliZangeneh, N. Nemati, Dr. B. Nikooyeh, M. Noormohammadi-Zarnagh, Dr. A. Nikoosaleh, F. Omid, S. Rajab, Dr. H. Rasekhi, N. Shariatzadeh, M. Zahehedi-Rad, F. Zakeri and T. Zowghi for their contribution in data collection and entry. The authors specially thank the head and staff of the local community of Tehransar (Naft-eShomali) and Taxi Organization of Tehran for their sincere helps during the study.

\section{Financial disclosure}

The authors declared no financial interest.

\section{References}

1. Lim SS, Vos T, Flaxman AD, Danaei G, Shibuya K, AdairRohani $\mathrm{H}$, et al. A comparative risk assessment of burden of disease and injury attributable to 67 risk factors and risk factor clusters in 21 regions, 1990-2010: a systematic analysis for the Global Burden of Disease Study 2010. The lancet. 2012;380(9859):2224-60. 
2. Popkin BM. The nutrition transition: an overview of world patterns of change. Nutrition reviews. 2004;62(suppl_2):S140-S3.

3. Afshin A, Micha R, Khatibzadeh S, Fahimi S, Shi P, Powles $\mathrm{J}$, et al. The impact of dietary habits and metabolic risk factors on cardiovascular and diabetes mortality in countries of the Middle East and North Africa in 2010: a comparative risk assessment analysis. BMJ open. 2015;5(5):e006385.

4. Clarke D, Herbert E. Food facts. London: Nelson. 1986:12-5.

5. Duffey KJ, Gordon-Larsen P, Jacobs Jr DR, Williams OD, Popkin BM. Differential associations of fast food and restaurant food consumption with 3-y change in body mass index: the Coronary Artery Risk Development in Young Adults Study. The American journal of clinical nutrition. 2007;85(1):201-8.

6. Maddock J. The relationship between obesity and the prevalence of fast food restaurants: state-level analysis. American journal of health promotion. 2004;19(2):137-43.

7. Pereira MA, Kartashov AI, Ebbeling CB, Van Horn L, Slattery ML, Jacobs Jr DR, et al. Fast-food habits, weight gain, and insulin resistance (the CARDIA study): 15-year prospective analysis. The lancet. 2005;365(9453):36-42.

8. Norton G, Anderson A, Hetherington M. Volume and variety: relative effects on food intake. Physiology \& Behavior. 2006;87(4):714-22.

9. Jon Schoenfeld B, Albert Aragon A, Krieger JW. Effects of meal frequency on weight loss and body composition: a metaanalysis. Nutrition reviews. 2015;73(2):69-82

10. Berg C, Lappas G, Wolk A, Strandhagen E, Torén K, Rosengren A, et al. Eating patterns and portion size associated with obesity in a Swedish population. Appetite. 2009;52(1):21-6.

11. Marín-Guerrero A, Gutiérrez-Fisac J, Guallar-Castillón P, Banegas J, Rodríguez-Artalejo F. Eating behaviours and obesity in the adult population of Spain. British journal of nutrition. 2008;100(5):1142-8.

12. Alhussain MH, Macdonald IA, Taylor MA. Irregular mealpattern effects on energy expenditure, metabolism, and appetite regulation: a randomized controlled trial in healthy normal-weight women. The American journal of clinical nutrition. 2016;104(1):21-32

13. Mozaffarian D, Appel LJ, Van Horn L. Components of a cardioprotective diet: new insights. Circulation. 2011;123(24):2870-91.

14. Amini M, Esmaillzadeh A, Omidvar N, Abtahi M, Dadkhah Piraghaj M, Nikooyeh B, et al. Development of a dish-based semi-quantitative food frequency questionnaire for Iranian population. Med J Islam Repub Iran 2019;(in press).

15. Doustmohammadian A, Amini M, Esmaillzadeh A, Omidvar N, Abtahi M, Dadkhah-Piraghaj $M$, et al. Validity and reliability of a dish-based semi-quantitative food frequency questionnaire for assessment of energy and nutrient intake among Iranian adults. BMC research notes. 2020;13(1):1-7.

16. Azar M, Sarkisian E. Food composition table of Iran. Tehran: National Nutrition and Food Research Institute, Shaheed Beheshti University. 1980;65.

17. Health NIo. Clinical guidelines for the identification, evaluation, and treatment of overweight and obesity in adultsthe evidence report. Obes Res. 1998;6(2):51S-209S.
18. Alberti KGMM, Zimmet P, Shaw J. International Diabetes Federation: a consensus on Type 2 diabetes prevention. Diabetic Medicine. 2007;24(5):451-63.

19. Buse JB, Ginsberg HN, Bakris GL, Clark NG, Costa F, Eckel $\mathrm{R}$, et al. Primary prevention of cardiovascular diseases in people with diabetes mellitus: a scientific statement from the American Heart Association and the American Diabetes Association. Circulation. 2007;115(1):114-26.

20. Kahleova H, Lloren JI, Mashchak A, Hill M, Fraser GE. Meal frequency and timing are associated with changes in body mass index in Adventist health study 2. The Journal of nutrition. 2017;147(9):1722-8.

21. Fabry P, Fodor J, Hejl Z, Braun T, Zvolánková K. The frequency of meals. Its relation to overweight, hypercholesterolaemia, and decreased glucose-tolerance. Lancet. 1964;2:614-5.

22. Ma Y, Bertone ER, Stanek III EJ, Reed GW, Hebert JR, Cohen NL, et al. Association between eating patterns and obesity in a free-living US adult population. American journal of epidemiology. 2003;158(1):85-92.

23. Metzner HL, Lamphiear DE, Wheeler N, Larkin FA. The relationship between frequency of eating and adiposity in adult men and women in the Tecumseh Community Health Study. The American journal of clinical nutrition. 1977;30(5):712-5.

24. Ruidavets J, Bongard V, Bataille V, Gourdy P, Ferrieres J. Eating frequency and body fatness in middle-aged men. International journal of obesity. 2002;26(11):1476.

25. Smeets AJ, Westerterp-Plantenga MS. Acute effects on metabolism and appetite profile of one meal difference in the lower range of meal frequency. British journal of nutrition. 2008;99(6):1316-21.

26. Speechly DP, Rogers GG, Buffenstein R. Acute appetite reduction associated with an increased frequency of eating in obese males. International journal of obesity. 1999;23(11):1151.

27. Bertelsen J, Christiansen C, Thomsen C, Poulsen PL, Vestergaard S, Steinov A, et al. Effect of meal frequency on blood glucose, insulin, and free fatty acids in NIDDM subjects. Diabetes care. 1993;16(1):4-7.

28. Jenkins D, Ocana A, Jenkins AL, Wolever T, Vuksan V, Katzman L, et al. Metabolic advantages of spreading the nutrient load: effects of increased meal frequency in noninsulin-dependent diabetes. The American journal of clinical nutrition. 1992;55(2):461-7.

29. Jenkins DJ, Wolever TM, Vuksan V, Brighenti F, Cunnane SC, Rao AV, et al. Nibbling versus gorging: metabolic advantages of increased meal frequency. New England Journal of Medicine. 1989;321(14):929-34.

30. LeBlanc J, Mercier I, Nadeau A. Components of postprandial thermogenesis in relation to meal frequency in humans. Canadian journal of physiology and pharmacology. 1993;71(12):879-83.

31. LeBlanc J, Diamond P. Effect of meal size and frequency on postprandial thermogenesis in dogs. American Journal of Physiology-Endocrinology and Metabolism. 1986;250(2):E144-E7.

32. Odegaard AO, Jacobs DR, Steffen LM, Van Horn L, Ludwig DS, Pereira MA. Breakfast Frequency and Development of Metabolic Risk. Diabetes Care. 2013;36(10):3100-6. 
33. Mekary RA, Giovannucci E, Willett WC, van Dam RM, Hu FB. Eating patterns and type 2 diabetes risk in men: breakfast omission, eating frequency, and snacking. The American journal of clinical nutrition. 2012;95(5):1182-9.

34. Holmbäck I, Ericson U, Gullberg B, Wirfält E. A high eating frequency is associated with an overall healthy lifestyle in middle-aged men and women and reduced likelihood of general and central obesity in men. British journal of nutrition. 2010;104(7):1065-73.

35. Edelstein SL, Barrett-Connor EL, Wingard DL, Cohn BA. Increased meal frequency associated with decreased cholesterol concentrations; Rancho Bernardo, CA, 19841987. The American Journal of Clinical Nutrition. 1992;55(3):664-9.

36. Saneei P, Esmaillzadeh A, Keshteli AH, Feizi A, FeinleBisset C, Adibi P. Patterns of dietary habits in relation to obesity in Iranian adults. European journal of nutrition. 2016;55(2):713-28.

37. Allison DB, Egan SK, Barraj LM, Caughman C, Infante M, T HEIMBACH J. Estimated intakes of trans fatty and other fatty acids in the US population. Journal of the American Dietetic Association. 1999;99(2):166-74.

38. Hulshof K, van Erp-Baart M, Anttolainen M, Becker W, Church S, Couet $C$, et al. Intake of fatty acids in western Europe with emphasis on trans fatty acids: the TRANSFAIR Study. European journal of clinical nutrition. 1999;53(2):143.

39. Mozaffarian D, Katan MB, Ascherio A, Stampfer MJ, Willett WC. Trans fatty acids and cardiovascular disease. New England Journal of Medicine. 2006;354(15):1601-13.

40. Willett WC, Stampfer MJ, Manson JE, Colditz GA, Speizer $\mathrm{FE}$, Rosner BA, et al. Intake of trans fatty acids and risk of coronary heart disease among women. The Lancet. 1993;341(8845):581-5.

41. Mauger J-F, Lichtenstein AH, Ausman LM, Jalbert SM, Jauhiainen M, Ehnholm C, et al. Effect of different forms of dietary hydrogenated fats on LDL particle size. The American journal of clinical nutrition. 2003;78(3):370-5.

42. Mensink RP, Zock PL, Kester AD, Katan MB. Effects of dietary fatty acids and carbohydrates on the ratio of serum total to HDL cholesterol and on serum lipids and apolipoproteins: a meta-analysis of 60 controlled trials. The American journal of clinical nutrition. 2003;77(5):1146-55.

43. Baer DJ, Judd JT, Clevidence BA, Tracy RP. Dietary fatty acids affect plasma markers of inflammation in healthy men fed controlled diets: a randomized crossover study. The American journal of clinical nutrition. 2004;79(6):969-73.
44. Han SN, Leka LS, Lichtenstein AH, Ausman LM, Schaefer EJ, Meydani SN. Effect of hydrogenated and saturated, relative to polyunsaturated, fat on immune and inflammatory responses of adults with moderate hypercholesterolemia. Journal of lipid research. 2002;43(3):445-52.

45. Lopez-Garcia E, Schulze MB, Meigs JB, Manson JE, Rifai N, Stampfer MJ, et al. Consumption of trans fatty acids is related to plasma biomarkers of inflammation and endothelial dysfunction. The Journal of nutrition. 2005;135(3):562-6.

46. Mozaffarian D, Pischon T, Hankinson SE, Rifai N, Joshipura $\mathrm{K}$, Willett WC, et al. Dietary intake of trans fatty acids and systemic inflammation in women. The American journal of clinical nutrition. 2004;79(4):606-12.

47. Clifton PM, Keogh JB. A systematic review of the effect of dietary saturated and polyunsaturated fat on heart disease. Nutr Metab Cardiovasc Dis. 2017;27(12):1060-80.

48. Hooper L, Martin N, Abdelhamid A, Davey Smith G. Reduction in saturated fat intake for cardiovascular disease. Cochrane Database Syst Rev. 2015(6):CD011737.

49. Cornée J, Pobel D, Riboli E, Guyader M, Hémon B. A casecontrol study of gastric cancer and nutritional factors in Marseille, France. European journal of epidemiology. 1995;11(1):55-65.

50. Ardekani MS, Salehi-Abargouei A, Mirzaei M, Fallahzadeh H, Nadjarzadeh A. Dietary habits in association with general and abdominal obesity in central Iran: Results from Yazd Health Study (YaHS). Diabetes \& Metabolic Syndrome: Clinical Research \& Reviews. 2018.

51. Bays HE, Toth PP, Kris-Etherton PM, Abate N, Aronne LJ, Brown WV, et al. Obesity, adiposity, and dyslipidemia: a consensus statement from the National Lipid Association. Journal of clinical lipidology. 2013;7(4):304-83.

52. Tabrizi R, Moosazadeh M, Akbari M, Dabbaghmanesh MH, Mohamadkhani M, Asemi Z, et al. High prevalence of vitamin $\mathrm{D}$ deficiency among Iranian population: a systematic review and meta-analysis. Iranian journal of medical sciences. 2018;43(2):125.

53. Mansouri M, Miri A, Varmaghani M, Abbasi R, Taha $P$, Ramezani S, et al. Vitamin D deficiency in relation to general and abdominal obesity among high educated adults. Eating and Weight Disorders-Studies on Anorexia, Bulimia and Obesity. 2019;24(1):83-90. 